\title{
Correlation of acetabular chondrocyte apoptosis with caspase-3 and Bcl-2 expression in developmental dislocations of the hip
}

\author{
L.J. Ding ${ }^{1 *}$, Y.L. Liu $^{2 *}$, G. Ma ${ }^{1}$, Y.Q. Jia ${ }^{3}$, Y.S.H. Wei ${ }^{1}$ and X.M. Liu ${ }^{1}$ \\ ${ }^{1}$ Department of Joint Surgery, \\ The Second Affiliated Hospital of Inner Mongolia Medical University, \\ Hohhot, Inner Mongolia, China \\ ${ }^{2}$ Rehabilitation Center, \\ The Second Affiliated Hospital of Inner Mongolia Medical University, \\ Hohhot, Inner Mongolia, China \\ ${ }^{3}$ Department of Integrated Medicine, \\ The Second Affiliated Hospital of Inner Mongolia Medical University, \\ Hohhot, Inner Mongolia, China \\ *These authors contributed equally to this study. \\ Corresponding authors: Y.S.H. Wei / X.M. Liu \\ E-mail: 3510803537@qq.com / kunchengibeijing@163.com
}

Genet. Mol. Res. 15 (3): gmr.15036809

Received May 15, 2015

Accepted May 19, 2016

Published September 23, 2016

DOI http://dx.doi.org/10.4238/gmr.15036809

Copyright (C) 2016 The Authors. This is an open-access article distributed under the terms of the Creative Commons Attribution ShareAlike (CC BY-SA) 4.0 License.

\begin{abstract}
This study aimed to determine whether abnormal apoptosis is present in acetabular cartilage in early developmental dislocations of the hip (DDH), and if so, whether it is correlated with the expression of caspase-3 and Bcl-2. DDH was induced in 24 4-week-old New Zealand white rabbits. Acetabular cartilage specimens from the experimental and control groups were stained with hematoxylin and eosin (H\&E). Animals from the experimental group developed acetabular dysplasia.
\end{abstract}


Apoptotic chondrocytes were observed by ultrastructural electron microscopy and H\&E. TUNEL assays revealed significantly greater acetabular chondrocyte apoptosis in the treated samples as compared to the control. Significantly higher caspase-3 expression and lower Bcl-2 expression were also measured in the DDH group compared with the control. We conclude that excessive apoptosis does occur in acetabular cartilage with DDH, and is positively correlated with high caspase-3 expression as well as low Bcl-2 expression.

Key words: Caspase-3; Bcl-2; Hip dislocation; Chondrocytes; Apoptosis

\section{INTRODUCTION}

The underlying cause of developmental dislocations of the hip (DDH) is not well characterized, and may be attributable to multiple factors. One such factor is articular cartilage degeneration, which has been reported in early DDH. In addition, secondary osteoarthritis (OA) has also been shown to occur with high incidence in late stages of the disease (Huang et al., 2013). The mechanisms by which acetabular cartilage degeneration occurs in early DDH is not yet clear. As apoptosis is an important mechanism in limb development, it is possible that defective apoptosis may contribute to articular cartilage degeneration in early DDH. How the expression of caspase- 3 and Bcl-2 is related to apoptosis and cartilage degeneration in DDH articular cartilage has also been a relatively unexplored area of research (Matsuo et al., 2001; Liu et al., 2014). This study attempted to determine whether abnormal apoptosis is present in early DDH acetabular cartilage, and if so, whether it is correlated with caspase-3 and Bcl-2 expression. To this end, differences in chondrocyte apoptosis as well as caspase-3 and Bcl2 expression were compared between control and treated conditions in New Zealand white rabbits with early DDH. Samples were analyzed by X-ray, visual anatomical observation, electron microscopy, and TUNEL assay.

\section{MATERIAL AND METHODS}

\section{Experimental animals, reagents, and devices}

\section{Experimental animals}

The 24 healthy 4-week-old New Zealand white rabbits weighing $0.5 \pm 0.05 \mathrm{~kg}$ (average weight $0.5 \mathrm{~kg}$ ) used in this experiment were provided to us by the Experimental Animal Center of Inner Mongolia Agricultural University.

\section{Immunohistochemistry reagents}

Rabbit anti-caspase-3 polyclonal antibody was provided by Beijing Sino-US Maixin Biomedical Technology Co., Ltd. Rabbit anti-Bcl-2 polyclonal antibody was provided by Wuhan Boster Biological Engineering Co., Ltd. The TUNEL assay kit was purchased from Wuhan Boster Biological Engineering Co., Ltd. The ready-to-use SP immunohistochemical staining kit was purchased from Beijing Sino-US Maixin Biomedical Technology Co., Ltd.

Genetics and Molecular Research 15 (3): gmr.15036809 


\section{Experimental methods and procedures}

\section{Animal modeling of $\mathrm{DDH}$}

All rabbits were anesthetized. The left lower limb was bent at the hip while keeping the knee straight using cylinder cast immobilization. The right lower limb was not treated, and served as the control. The rabbits were singly housed under standard feeding conditions in the Animal Care Center of Inner Mongolia Medical University. They were examined by X-ray after 8 weeks to select 24 animals with DDH, as judged by the acetabular index, Shenton's line, and location of the left femoral head in the lateral superior and inferior quadrants of Perkin's square. The 24 rabbits with DDH were randomly divided into two groups of 12 . One group was immediately sacrificed while the other was housed with cast immobilization for another 2 weeks prior to sacrifice.

\section{Acetabulum sample preparation}

The hip was immediately dissected following sacrifice. Morphological changes in the hip were observed and photographed after capsular incision was made in the femoral neck. In preparation for transmission electron microscopy imaging, 1- $\mathrm{mm}^{3}$ cartilage samples were resected from the posterosuperior acetabulum. TUNEL staining and immunohistochemistry were also performed on control and treated samples. Histological structures were compared between the experimental and control sides after paraffin embedding and staining with hematoxylin and eosin (H\&E).

\section{TUNEL assay}

TUNEL assay was performed according to manufacturer specifications. The biotinylated anti-digoxin antibody was diluted 1:100 in antibody diluent, and added to the sections at 501:100/section. The sections were then incubated with $1 \mathrm{~mL} 0.01 \mathrm{M}$ TBS and 10 $\mu \mathrm{L}$ StreptAvidin-Biotin Complex. Lastly, sections were developed with 3,3'-diaminobenzidine (DAB), stained with hematoxylin, and mounted with neutral resin.

\section{SP immunohistochemistry}

Paraffin-embedded sections were dewaxed, and rehydrated using an alcohol gradient. This was followed by heat-induced antigen retrieval. Blocking of endogenous peroxidase activity and non-specific binding was performed in a mixture containing $50 \mu \mathrm{L}$ solution A (peroxidase blocking solution) and $50 \mu \mathrm{L}$ solution B (normal non-immune serum). Sections were incubated with appropriately diluted primary antibodies (caspase- 3 and Bcl-2 antibodies were both diluted), then developed with DAB (2 min). Stained sections were visualized under an optical microscope.

\section{Transmission electron microscopy}

\section{Quality control and judgment of positivity}

Detection of chondrocyte apoptosis was analyzed by TUNEL assay. TUNELpositive cells contained brown cytoplasm or membrane, as visualized by light microscopy.

Genetics and Molecular Research 15 (3): gmr.15036809 
Chondrocytes with brown granules within the nucleus were therefore classified as apoptotic cells. Images were converted to grayscale, and average intensity of Bcl-2 and caspase-3 were determined using a pathological image analysis system for semi-quantitative analysis; the greater the grayscale intensity, the lower the positive cytoplasmic expression of the factor, and vice versa.

\section{Data analysis}

All data are reported as means $\pm \mathrm{SD}$, and imported to SPSS 18.0 for statistical analysis. The acetabular index, apoptosis rate, and grayscale values were compared between the experimental and control groups using the paired sample $t$-test with $\alpha=0.05$. $\mathrm{P}<0.05$ was considered to be statistically significant. Correlation of chondrocyte apoptosis with caspase-3 and Bcl-2 was also analyzed.

\section{RESULTS}

\section{Gross morphology}

\section{Control side}

The hip capsule had uniform thickness and good tension. The acetabular head was normal in contour. Separation between the acetabulum and the femoral head was only possible with the opening of the joint capsule due to high acetabular femoral head coverage and tight connection. There was significant intra-articular negative pressure and an appropriate amount of articular synovia. The acetabular cartilage had a smooth and glossy surface, with no impression. The acetabulum had a round shape, with uniform rim thickness and a deep fossa. The round ligament had an appropriate length (Figures 1 and 2).

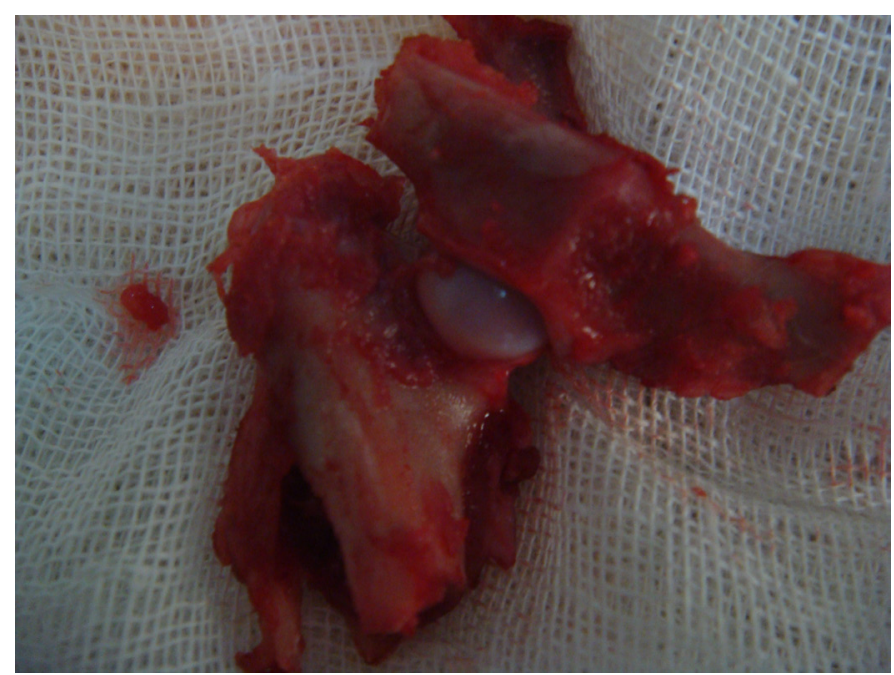

Figure 1. High acetabular coverage of the femoral head in the opened joint capsule. 


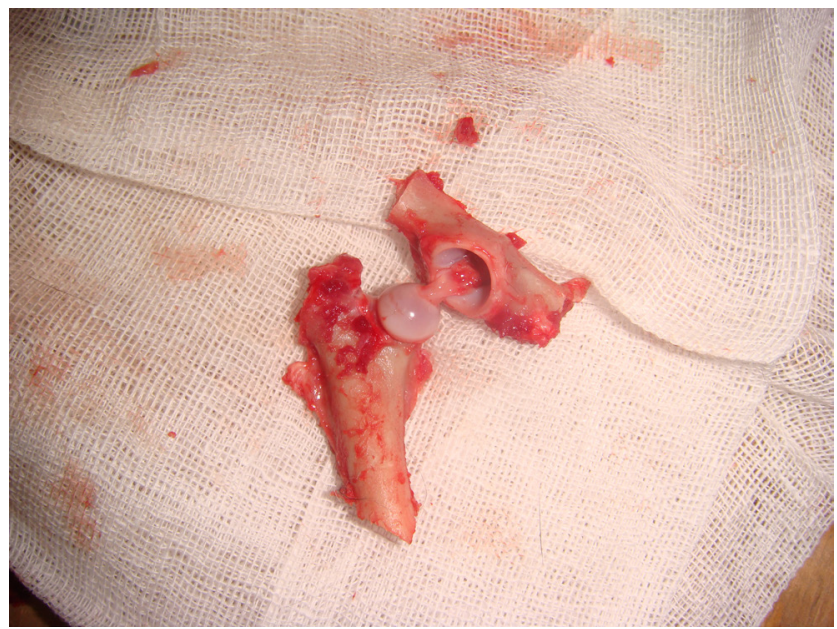

Figure 2. Normal contour of acetabulum and femoral head with smooth surface of acetabular cartilage, and appropriate length of round ligament in the control side.

\section{Experimental side}

The following general acetabular changes were observed after 8 weeks of immobilization: thickened joint capsule; loose joint that was easy to dislocate by manipulation (no longer round in contour); impression on the posterosuperior lateral rim of the acetabulum in most cases; shallow acetabulum poorly covering the femoral head; dislocated femoral head; dull surface of the articular cartilage; a large amount of articular synovia; flattened and shrunken femoral head; loose joint capsule and round ligament (Figures 3 and 4).

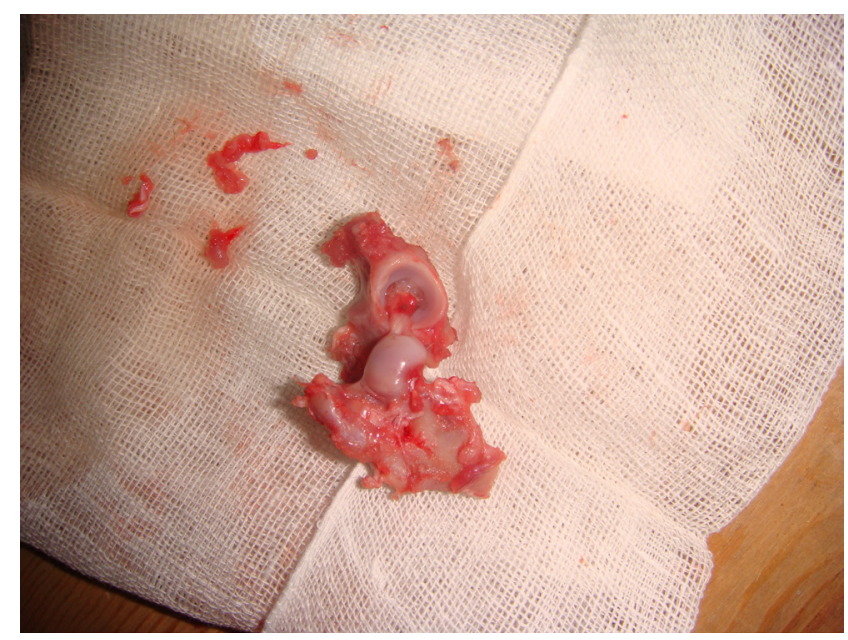

Figure 3. Loose joint that was no longer round but oval in contour, the presence of impressions on the posterosuperior lateral rim of the acetabulum, and flattened and shrunken femoral head.

Genetics and Molecular Research 15 (3): gmr.15036809 


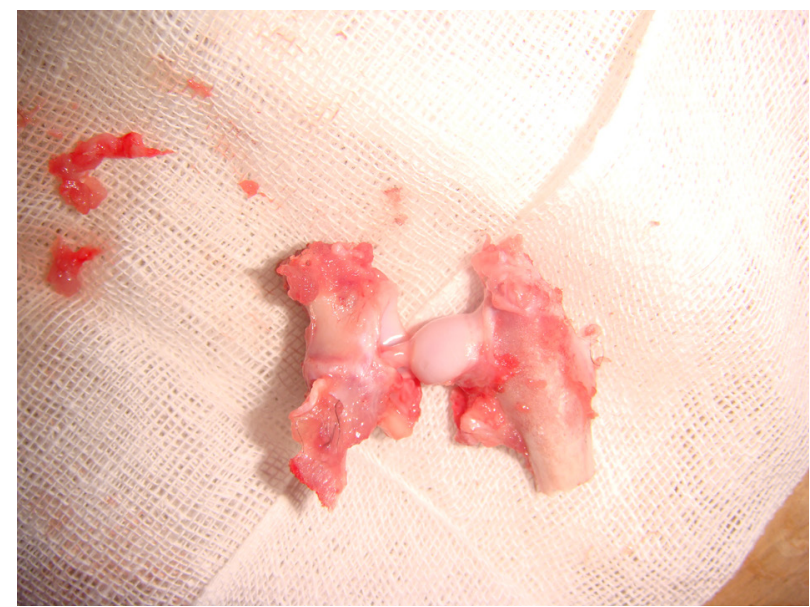

Figure 4. Loose joint capsule and round ligament, thickened joint capsule, easily dislocated joint, and dull surface of the articular cartilage.

The following general acetabular changes were observed after 10 weeks of immobilization: significantly changed contour and abnormal anatomy of the acetabulum; significantly loose, deformed, and thickened joint capsule; hypertrophy and introversion of the glenoid labrum; a small amount of muddy articular synovia; long narrow flat dysplastic acetabulum filled with soft tissue; a deep narrow inner acetabulum and a shallow flat outer acetabulum; hyperplasia of the posterosuperior acetabulum; widened and flattened acetabular rim; rough surface of the posterosuperior cartilage resulting from abrasion against the femoral head; ulcers of parts of the posterosuperior acetabulum due to cartilage desquamation; yellowcolored cartilage with poor flexibility; hip instability; lateral superior dislocation of the femoral head; decreased size of the dislocated femoral head; stretching and hypertrophy of the round ligament (Figures 5 and 6).

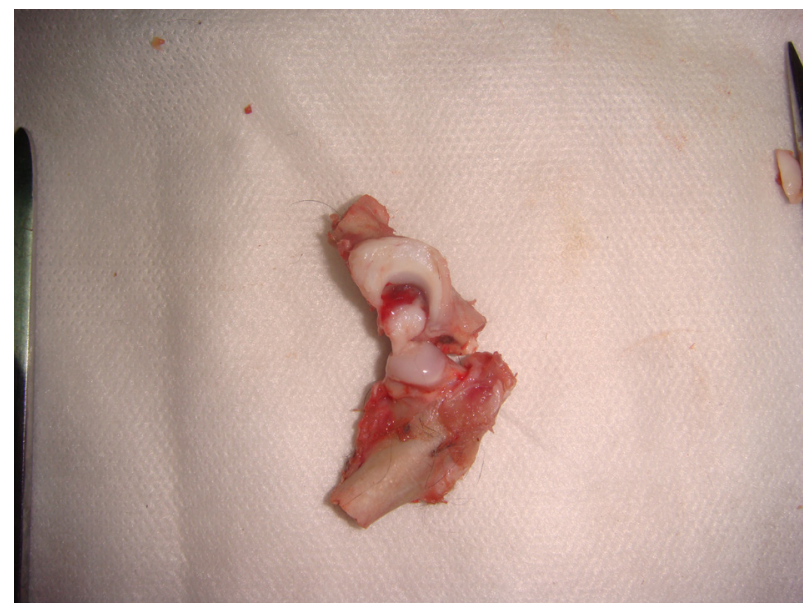

Figure 5. Flat acetabulum, dysplasia of the femoral head, low congruency between the femoral head and acetabulum, and dull yellow-colored cartilage.

Genetics and Molecular Research 15 (3): gmr.15036809 


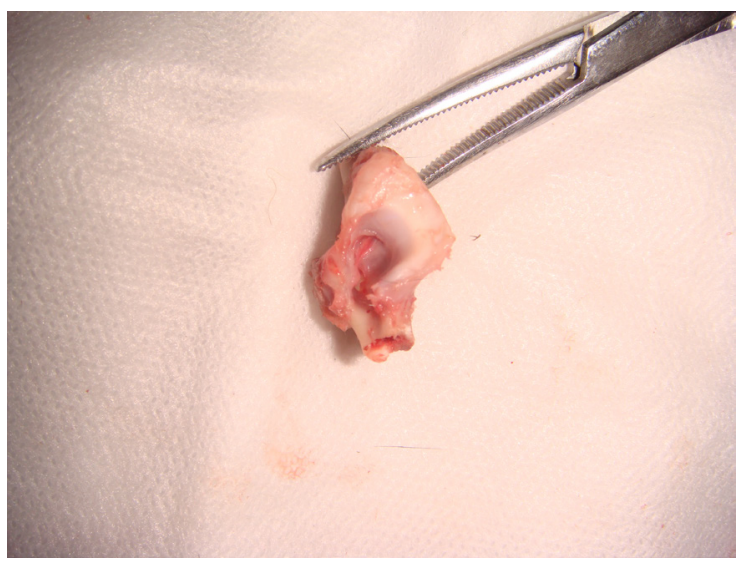

Figure 6. Long narrow dysplastic acetabulum with abnormal anatomy, a deep narrow inner acetabulum and a shallow flat outer acetabulum, serious abrasion against the posterosuperior articular surface, and uneven surface of the posterosuperior cartilage.

\section{Pelvic X-ray observations}

For the control side of the hip, a normal structure was observed with high congruency between the femoral head and acetabulum, a clear contour, and a smooth articular surface (Figures 7 and 8). For the experimental side of the hip, acetabular dysplasia was observed, manifesting as a blunt, straight, and uptilted upper rim of the acetabulum. The femoral head was shrunken, with decreased acetabular coverage, broken Shenton's line, and a dislocation of the left femoral head to the lateral superior or inferior quadrant of Perkin's square. A higher degree and incidence of subluxation and dislocation was observed in the 10-week group compared with the 8-week group (Figures 8 and 9).
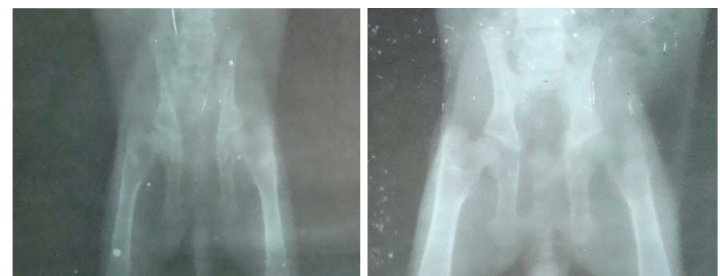

Figure 7. X-ray image of normal hips before immobilization: normal structure of bilateral hips, and high congruency between the femoral head and acetabulum.
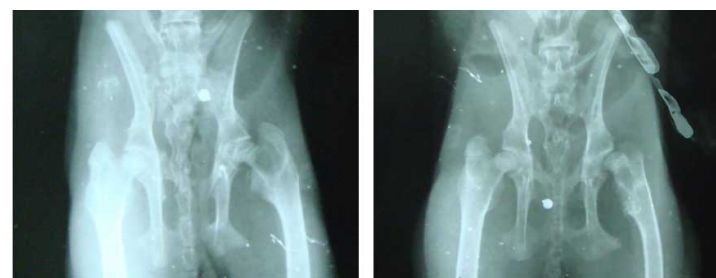

Figure 8. X-ray image of the experimental (left) hip after 8 weeks of immobilization: acetabular dysplasia, upturned upper rim of the acetabulum, and subluxation of the femoral head to the lateral superior quadrant.

Genetics and Molecular Research 15 (3): gmr.15036809 

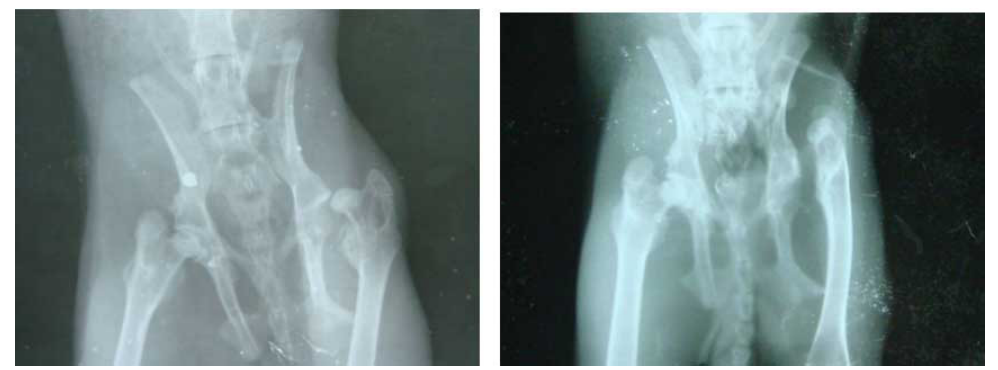

Figure 9. X-ray image of the experimental (left) hip after 10 weeks of immobilization: hip dislocation, shallow acetabulum, decreased acetabular coverage of the femoral head, blunt, straight, and even uptilted upper rim of the acetabulum.

\section{Determination and comparison of acetabular index}

A significant difference was noted in the acetabular index between the experimental and control sides in both the 8-week group $(\mathrm{P}<0.05)$ and the 10 -week group $(\mathrm{P}<0.01)$. No difference was observed in the acetabular index in the control side before and after immobilization of the experimental side $(\mathrm{P}>0.05)$ (Table 1 and Figure 10).

Table 1. Change in the acetabular index (degree) in the experimental and control sides (means $\pm \mathrm{SD}$ ).

\begin{tabular}{l|c|c|c|c|c}
\hline Group & $\mathrm{N}$ & $\begin{array}{c}\text { Before 8-week } \\
\text { immobilization }\end{array}$ & $\begin{array}{c}\text { After 8-week } \\
\text { immobilization }\end{array}$ & $\begin{array}{c}\text { Before 10-week } \\
\text { immobilization }\end{array}$ & $\begin{array}{c}\text { After 10-week } \\
\text { immobilization }\end{array}$ \\
\hline Experimental & 12 & $24.42 \pm 1.39^{*}$ & $27.00 \pm 1.21^{\Delta}$ & $24.63 \pm 1.60^{*}$ & $24.29 \pm 1.46^{*}$ \\
\hline Control & 12 & $24.38 \pm 1.56^{*}$ & $25.37 \pm 2.05^{\Delta}$ & $29.43 \pm 2.45^{\mathbf{\Delta}}$ & $25.26 \pm 1.66^{\mathbf{\Delta}}$ \\
\hline
\end{tabular}

$* \mathrm{P}>0.05, \triangle \mathrm{P}<0.05,{ }^{\Delta} \mathrm{P}<0.01$ between the experimental and control sides.

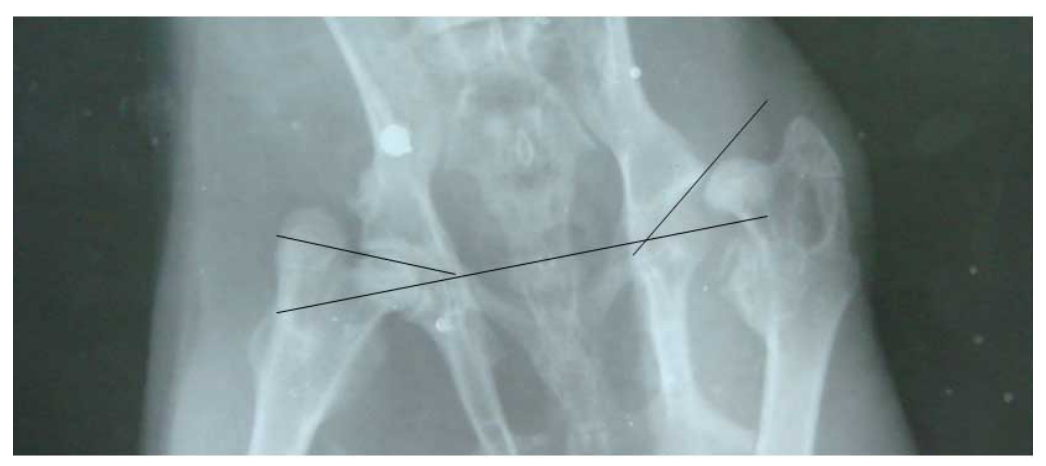

Figure 10. Measurement of acetabular index.

\section{Pathological examination by H\&E staining}

The H\&E-stained acetabular chondrocytes were found to have a regular morphology. They were densely and uniformly distributed, are oblate in shape, and are located in the cartilage lacuna. In addition, they contain light colored nuclei that are evenly distributed with clearly visible nucleoli (Figures 11 and 12). 

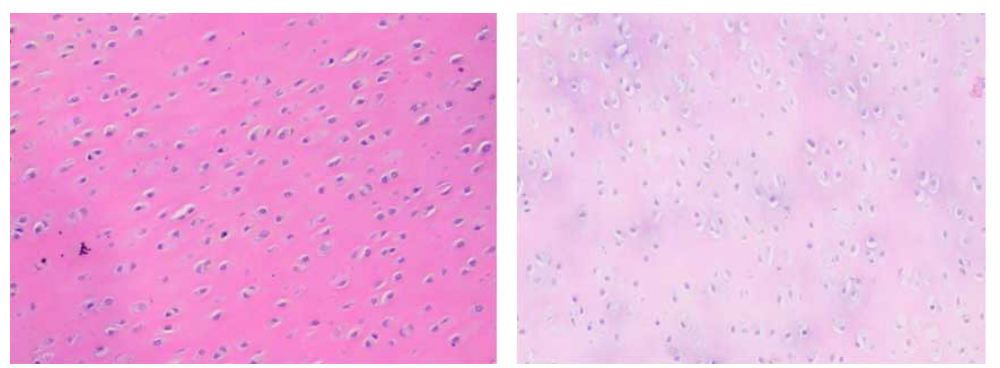

Figure 11. Regular morphology and oblate shape of acetabular chondrocytes located in the cartilage lacuna, which contain light colored nucleus with distinct and regularly distributed nucleolus (H\&E, 400X).
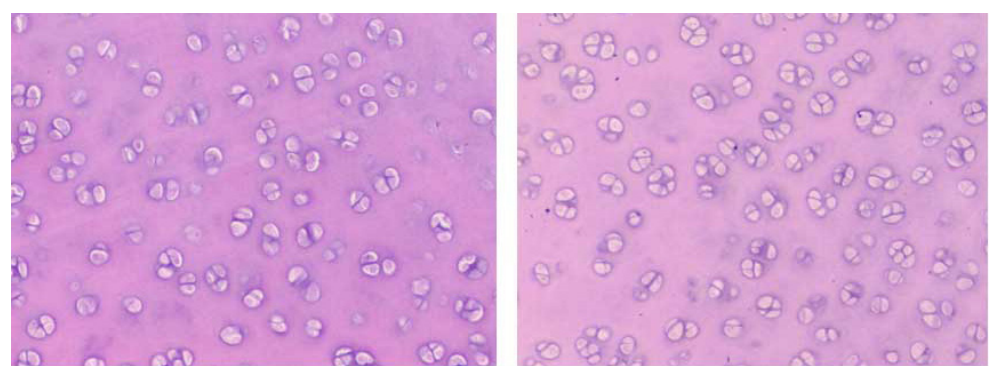

Figure 12. Chondrocytes in the experimental side: sparse and irregular in distribution, abnormal morphology, increased vacuolar cells, increased volume of vacuoles, gathering in clusters, and increased blank area (H\&E, 400X).

\section{Observation by electron microscopy}

For the control side, uniformly distributed acetabular chondrocytes with regular morphology and clearly visible organelles were observed (Figure 13). After 8 weeks of immobilization, decreased chondrocytes were seen with irregular morphology, chromatin margination and condensation, increased electron density and nucleus density, and nucleus condensation. These changes were more prominent after 10 weeks of immobilization. Furthermore, the organelles were scattered and difficult to identify, and the cytoplasm contained many vacuoles (i.e., cavitation) (Figure 14).
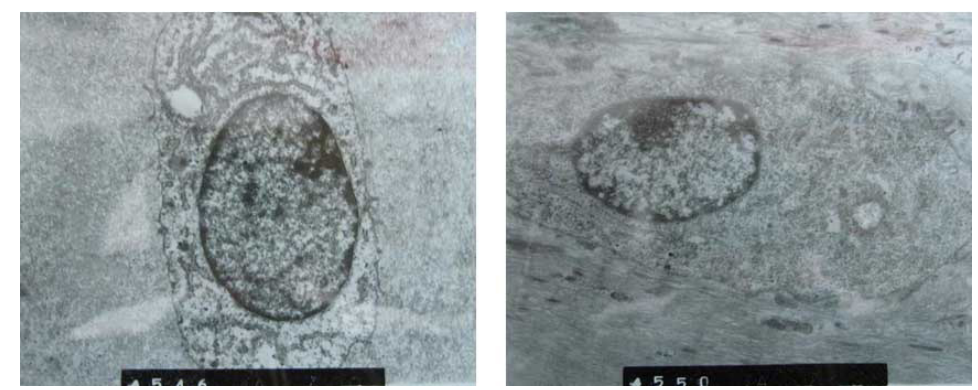

Figures 13. Chondrocytes in the control side show clearly visible organelles, regular morphology of the nucleus, and normal morphology of the mitochondria and endoplasmic reticulum (9000X).

Genetics and Molecular Research 15 (3): gmr.15036809 

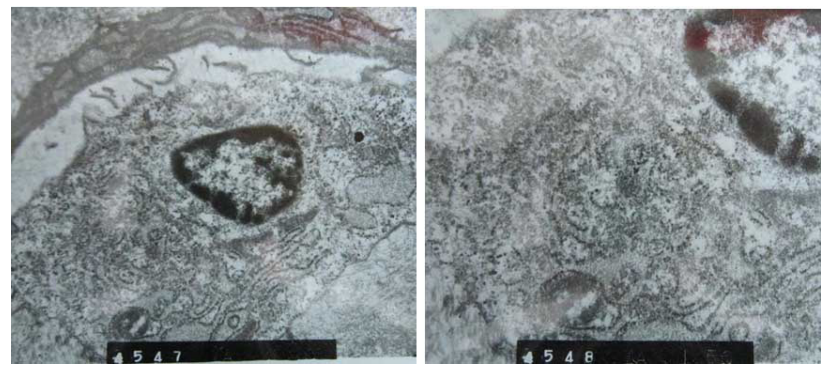

Figures 14. Chondrocytes in the experimental side display irregular morphology, nuclear changes and aggregation of matrix particles, increased density and condensation of nucleus, numerous vacuoles in the cytoplasm, glycoprotein-rich matrix with very little amount of collagen, coagulation of matrix-depleted cytoplasm into small granules, vacuolar mitochondria, and poorly identifiable organelles (9000X).

\section{Detection of apoptosis by TUNEL assay}

Chondrocytes with brown particles in the nucleus were apoptotic cells, whereas nuclei of normal chondrocytes appeared blue following hematoxylin staining. Apoptotic cells were rarely seen in specimens obtained from the control side (Figure 15). Significantly increased apoptotic chondrocytes were observed at the experimental side compared with the control side (Figure 16). A significant difference was noted between the experimental and control sides in both the 8-week group $(\mathrm{P}<0.05)$ and the 10 -week group $(\mathrm{P}<0.01)$, as quantified by the paired $t$-test. No significant difference was noted in the apoptosis rate of acetabular chondrocytes between the 8- and 10-week control groups $(\mathrm{P}>0.05)$ or between the 8-and 10week experimental groups $(\mathrm{P}>0.05)$ (Tables 2, 3, 4, and 5).

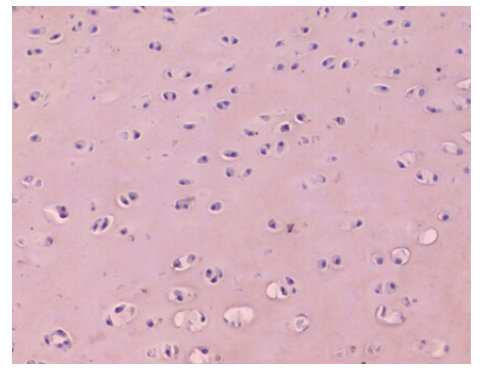

Figure 15. Rare yellow-stained apoptotic nuclei in chondrocytes in the control side (H\&E, 400X).

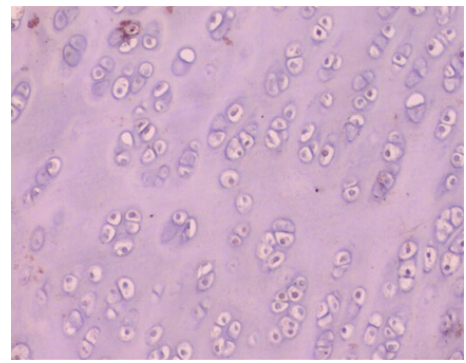

Figure 16. Yellow-stained apoptotic nuclei in chondrocytes in the experimental side (H\&E, 400X).

Genetics and Molecular Research 15 (3): gmr.15036809 
Table 2. Comparison of apoptosis between the 8-week experimental and control groups.

\begin{tabular}{l|c|c|c|c}
\hline Group & $\mathrm{N}$ & Apoptosis rate (\%) & $t$-value & P value \\
\hline Experimental & 12 & $0.045 \pm 0.013$ & 8.442 & 0.000 \\
\hline Control & 12 & $0.008 \pm 0.007$ & & \\
\hline
\end{tabular}

Table 3. Comparison of apoptosis between the 10-week experimental and control groups.

\begin{tabular}{l|c|c|c|c}
\hline Group & $\mathrm{N}$ & Apoptosis rate (\%) & $t$-value & P value \\
\hline Experimental & 12 & $0.050 \pm 0.019$ & 6.156 & 0.000 \\
\hline Control & 12 & $0.012 \pm 0.010$ & & \\
\hline
\end{tabular}

Table 4. Comparison of apoptosis between the 8- and 10-week control groups.

\begin{tabular}{l|c|c|c|c}
\hline Group & $\mathrm{N}$ & Apoptosis rate (\%) & $t$-value & P value \\
\hline 8-week control & 12 & $0.008 \pm 0.007$ & 0.369 & 0.716 \\
\hline 10-week control & 12 & $0.012 \pm 0.010$ & & \\
\hline
\end{tabular}

Table 5. Comparison of apoptosis between the 8- and 10-week experimental groups.

\begin{tabular}{l|c|c|c|c}
\hline Group & $\mathrm{N}$ & Apoptosis rate (\%) & $t$-value & P value \\
\hline 8-week experimental & 12 & $0.045 \pm 0.013$ & 0.701 & 0.491 \\
\hline 10-week experimental & 12 & $0.050 \pm 0.019$ & & \\
\hline
\end{tabular}

\section{Detection of caspase-3 and Bcl-2 by immunohistochemistry}

\section{Caspase-3 expression in the acetabular cartilage cells}

Caspase- 3 expression was detected in the acetabular chondrocytes after 8 weeks of immobilization in both the control and experimental sides, while a significantly lower mean grayscale value was measured in the experimental group compared with the control group ( $\mathrm{P}$ $<0.05$ ) (Table 6 and Figures 17 and 18).

Table 6. Comparison of caspase- 3 expression in the acetabular chondrocytes between the 8 -week experimental and control groups.

\begin{tabular}{l|c|c|c}
\hline Group & Mean grayscale value & $t$-value & P value \\
\hline Experimental & $56.73 \pm 7.85$ & 2.420 & 0.020 \\
\hline Control & $61.45 \pm 5.47$ & & \\
\hline
\end{tabular}

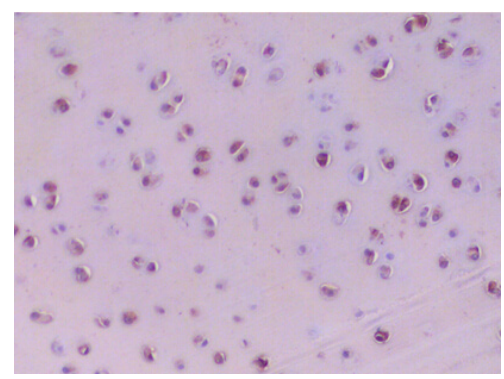

Figure 17. Caspase-3 expression in chondrocytes in the control side after 8 weeks of immobilization (H\&E, 400X).

Genetics and Molecular Research 15 (3): gmr.15036809 


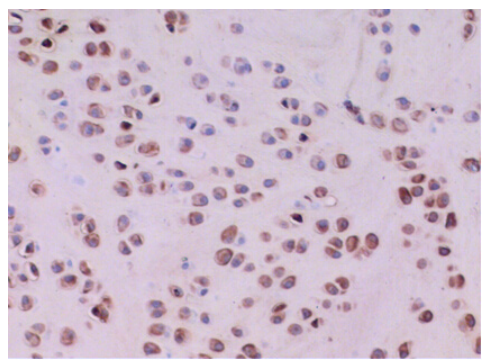

Figure 18. Higher caspase-3 expression in chondrocytes in the experimental side compared to the control side after 8 weeks of immobilization (H\&E, 400X).

Similarly, caspase-3 expression was also detected in the acetabular chondrocytes after 10 weeks of immobilization in both the control and experimental sides, while a significantly lower mean grayscale value was measured in the experimental group compared with the control group $(\mathrm{P}<0.01)$ (Table 7 and Figures 19 and 20).

No significant difference was noted in the mean grayscale value of caspase- 3 expression between the 8 - and 10 -week control groups $(\mathrm{P}>0.05$; Table 8$)$ or between the 8 and 10-week experimental groups $(\mathrm{P}>0.05$; Table 9).

Table 7. Comparison of caspase- 3 expression in the acetabular chondrocytes between the 10 -week experimental and control groups.

\begin{tabular}{l|c|c|c}
\hline Group & Mean grayscale value & $t$-value & P value \\
\hline Experimental & $56.48 \pm 7.94$ & 2.834 & 0.007 \\
\hline Control & $62.12 \pm 5.67$ & & \\
\hline
\end{tabular}

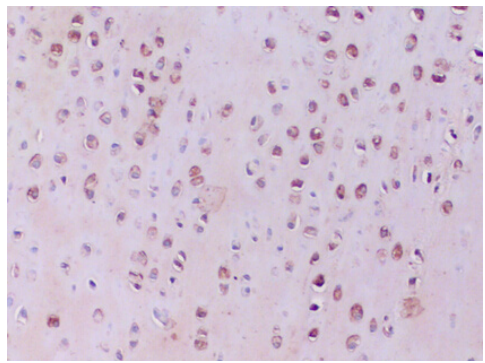

Figure 19. Caspase-3 expression in chondrocytes in the control side after 10 weeks of immobilization (H\&E, 400X).

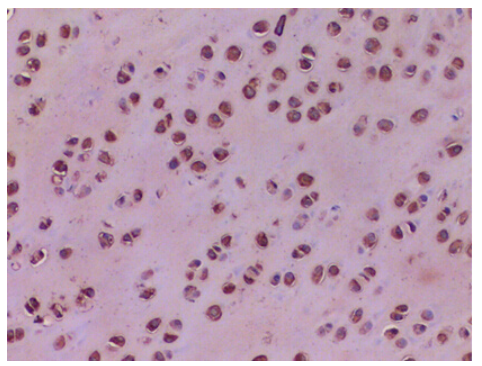

Figure 20. Significantly higher caspase-3 expression in chondrocytes in the experimental side compared to the control side after 10 weeks of immobilization (H\&E, 400X).

Genetics and Molecular Research 15 (3): gmr.15036809 
Table 8. Comparison of caspase-3 expression in the acetabular chondrocytes between the 8- and 10-week control groups.

\begin{tabular}{l|c|c|c}
\hline Group & Mean grayscale value & $t$-value & P value \\
\hline 8 -week control & $61.45 \pm 5.47$ & -0.415 & 0.680 \\
\hline 10 -week control & $62.12 \pm 5.67$ & & \\
\hline
\end{tabular}

Table 9. Comparison of caspase-3 expression in the acetabular chondrocytes between the 8- and 10-week experimental groups.

\begin{tabular}{l|c|c|c}
\hline Group & Mean grayscale value & $t$-value & P value \\
\hline 8-week experimental & $56.73 \pm 7.85$ & 0.110 & 0.913 \\
\hline 10-week experimental & $56.48 \pm 7.94$ & & \\
\hline
\end{tabular}

Bcl-2 expression was detected in the acetabular chondrocytes after 8 weeks of immobilization in both the control and experimental sides, while a significantly lower mean grayscale value was measured in the experimental group compared with the control group (P $<0.05$ ) (Table 10 and Figures 21 and 22).

Table 10. Comparison of Bcl-2 expression in the acetabular chondrocytes between the 8-week experimental and control groups.

\begin{tabular}{l|c|c|c}
\hline Group & Mean grayscale value & $t$-value & P value \\
\hline Experimental & $58.31 \pm 10.06$ & -2.285 & 0.027 \\
\hline Control & $52.32 \pm 7.99$ & & \\
\hline
\end{tabular}

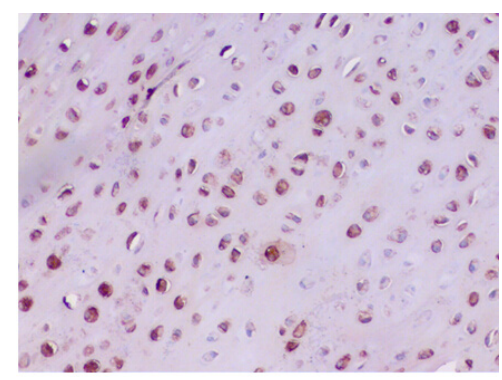

Figure 21. Bcl-2 expression in chondrocytes in the control side after 8 weeks of immobilization (H\&E, 400X).

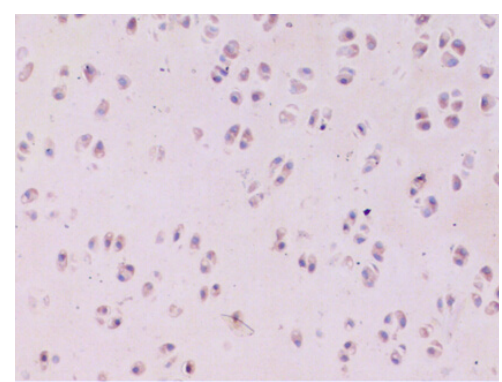

Figure 22. Lower Bcl-2 expression in chondrocytes of the experimental side compared to the control side after 8 weeks of immobilization (H\&E, 400X).

Genetics and Molecular Research 15 (3): gmr.15036809 
Similarly, Bcl-2 expression was detected in the acetabular chondrocytes after 10 weeks of immobilization in both the control and experimental sides, while a significantly lower mean grayscale value was measured in the experimental group compared with the control group ( $\mathrm{P}$ $<0.01$ ) (Table 11 and Figures 23 and 24).

No significant difference was noted in the mean grayscale value of Bcl-2 expression between the 8 - and 10 -week control groups $(\mathrm{P}>0.05$; Table 12) or between the 8 - and 10-week experimental groups $(\mathrm{P}>0.05$; Table 13).

Table 11. Comparison of Bcl-2 expression in the acetabular chondrocytes between the 10 -week experimental and control groups.

\begin{tabular}{l|c|c|c}
\hline Group & Mean grayscale value & $t$-value & P value \\
\hline Experimental & $59.73 \pm 9.16$ & -3.210 & 0.002 \\
\hline Control & $51.90 \pm 7.66$ & & \\
\hline
\end{tabular}

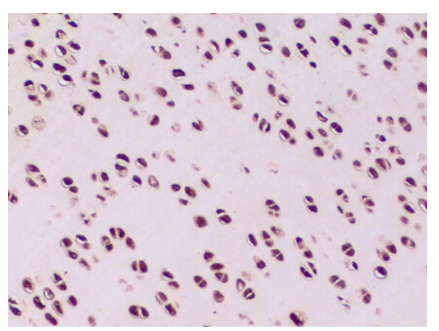

Figure 23. Bcl-2 expression in chondrocytes of the control side after 10 weeks of immobilization (H\&E, 400X).

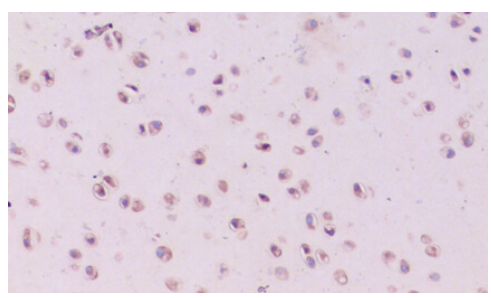

Figure 24. Significantly decreased Bcl-2 expression in chondrocytes in the experimental side compared to the control side after 10 weeks of immobilization (400X).

Table 12. Comparison of Bcl-2 expression in the acetabular chondrocytes between the 8- and 10-week control groups.

\begin{tabular}{l|c|c|c}
\hline Group & Mean grayscale value & $t$-value & P value \\
\hline 8-week control & $52.32 \pm 7.99$ & 0.184 & 0.854 \\
\hline 10-week control & $51.90 \pm 7.66$ & & \\
\hline
\end{tabular}

Table 13. Comparison of Bcl-2 expression in the acetabular chondrocytes between the 8 - and 10 -week experimental groups.

\begin{tabular}{l|c|c|c}
\hline Group & Mean gray value & $t$-value & P value \\
\hline 8-week experimental & $58.31 \pm 10.06$ & -0.510 & 0.612 \\
\hline 10-week experimental & $59.73 \pm 9.16$ & & \\
\hline
\end{tabular}

Genetics and Molecular Research 15 (3): gmr.15036809 


\section{Correlation of chondrocyte apoptosis with caspase-3 and Bcl-2}

Caspase-3 and Bcl-2 expression was positively and negatively correlated with chondrocyte apoptosis, respectively (Figures 25, 26, 27, and 28).

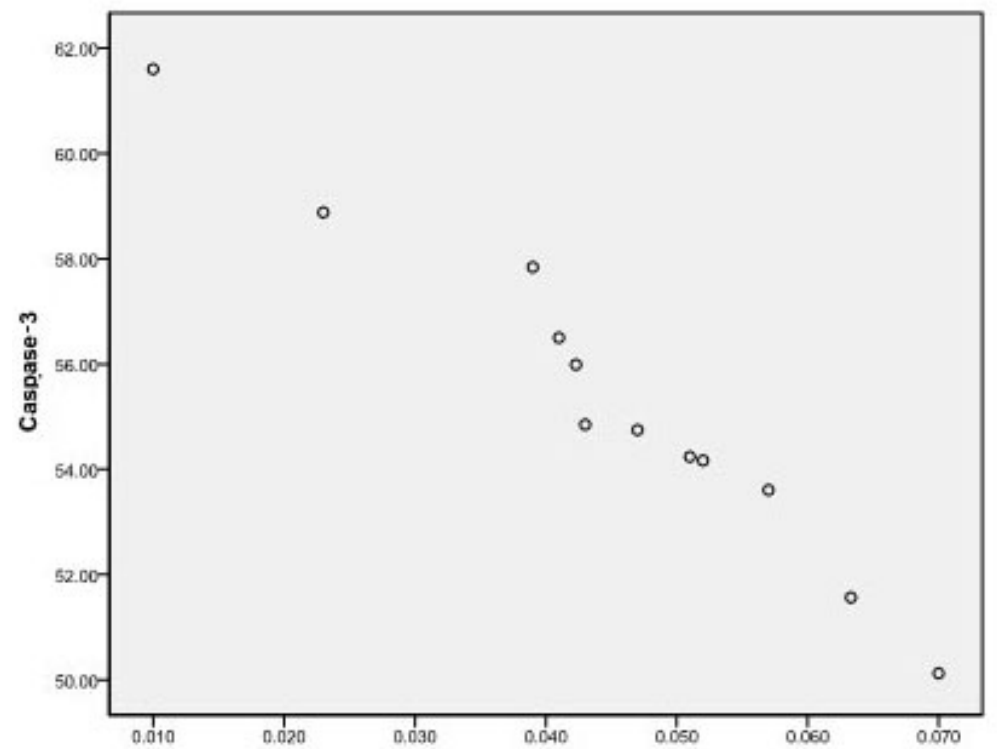

Figure 25. Correlation of caspase-3 grayscale value with apoptosis in the 8-week experimental side.

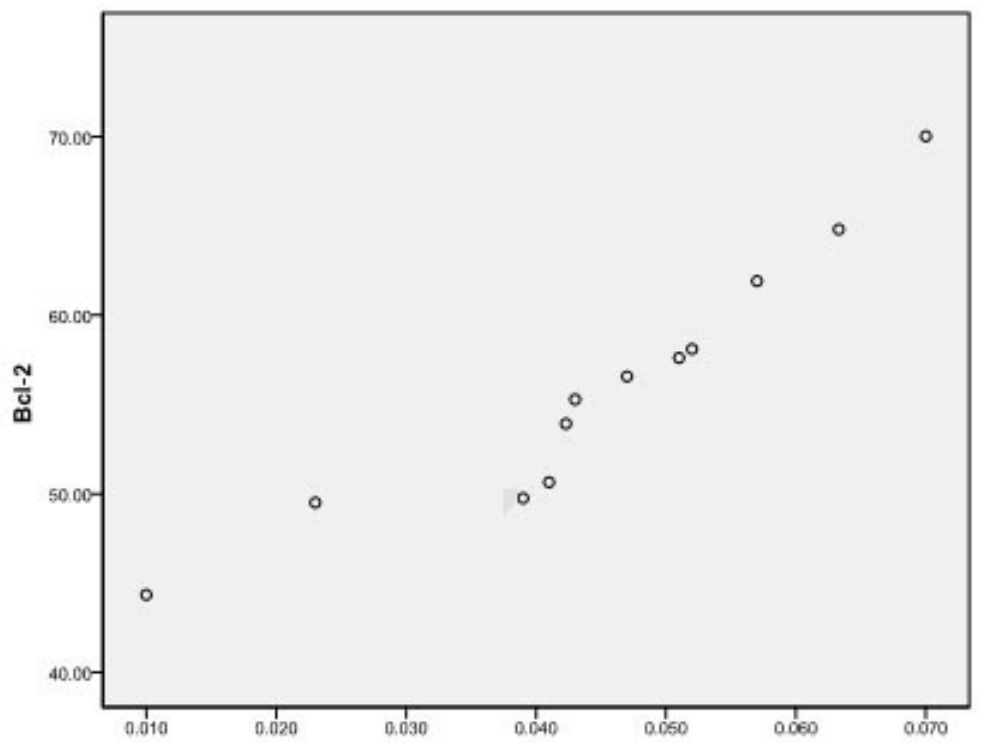

Figure 26. Correlation of Bcl-2 grayscale value with apoptosis in the 8-week experimental side.

Genetics and Molecular Research 15 (3): gmr.15036809 


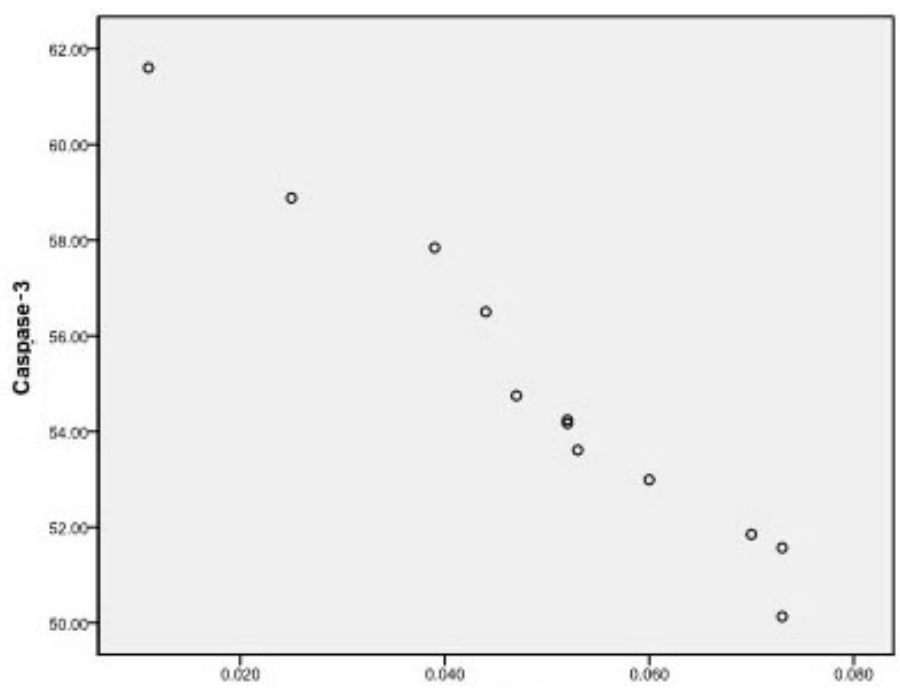

Figure 27. Correlation of caspase-3 grayscale value with apoptosis in the 10-week experimental side.

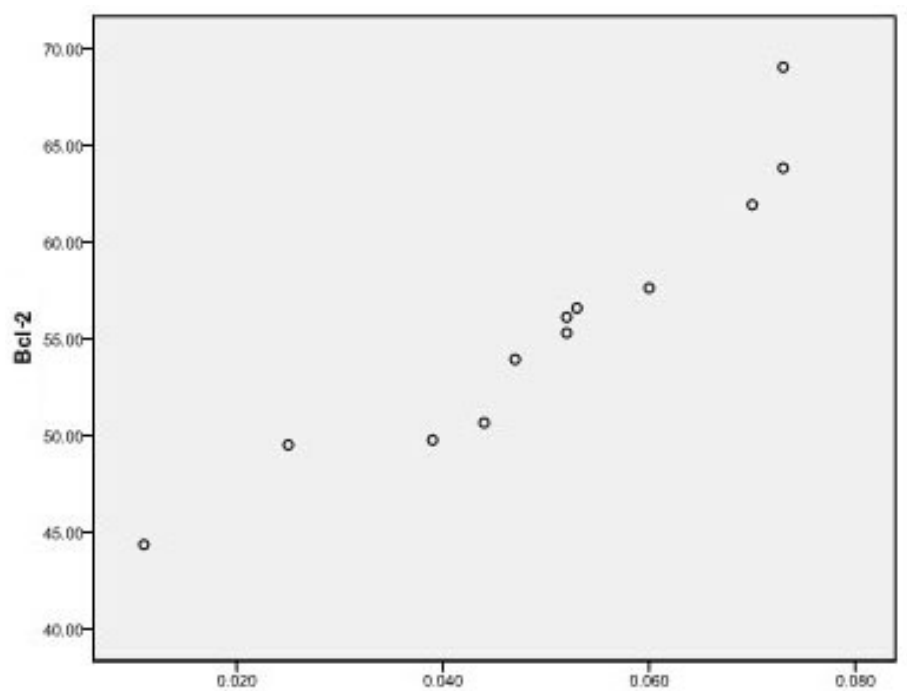

Figure 28. Correlation of Bcl-2 grayscale value with apoptosis in the 10-week experimental side.

\section{DISCUSSION}

\section{DDH modeling}

Animal models are essential for studies of human diseases. In this study, early DDH was developed in New Zealand white rabbits. It was reported that rabbits less than 10 weeks old were biological equivalents to approximately 7- to 9-year-old children, which is a useful model for early stage of DDH (Greenhill et al., 1995). We developed a model of early DDH in 
rabbits via 8 - and 10-week immobilization. Immobilization was performed using polymer plaster based on reported studies and previous experience. The holes of the polymer plaster itself can provide decompression. Moreover, the tube-shaped connection is not permanently glued, which allows for flexibility and avoids excessive plaster, thus providing decompression. The polymer plaster is similar to chamotte. It has a smooth surface, which makes it unlikely to adhere to rabbit fur, and is easy to remove and replace. Hence, the plaster was easily replaced for debonding or re-immobilization approximately every 3 weeks. After 8 weeks of immobilization, DDH had developed in 24 rabbits, as revealed by X-ray imaging. The 24 rabbits with DDH were then randomly divided into two groups of 12, which were sacrificed at week 8 and 10 . Changes related to acetabular dysplasia were determined by $\mathrm{X}$-ray imaging and visual anatomical observation.

\section{DDH and cartilage degeneration}

Several studies have shown that acetabular dysplasia, which occurs in DDH, results from the combined effects of genetic factors, abnormal mechanical stress, and hormonal factors. The following changes were observed in chondrocytes of the experimental side by H\&E staining: sparse and irregular distribution, cluster formation, abnormal morphology, increased vacuolar cells, increased volume of vacuoles, and increased blank area. These observations present evidence of cartilage degeneration in acetabular cartilage specimens in the experimental group. Moreover, these morphological changes became much more pronounced with extension of the course of DDH. We have demonstrated that early DDH does involve cartilage degeneration and may lead to development of OA.

\section{Acetabular cartilage lesion and apoptosis in DDH}

Only few studies have been conducted to investigate whether apoptosis occurs and the proportion of apoptotic cells in chondrocytes in DDH-related acetabular dysplasia (Pelletier et al., 2000; Jiang et al., 2014). In this study, increased apoptosis of chondrocytes was detected in DDH articular cartilage tissue sections using a TUNEL assay. In addition, the following changes were observed in the experimental side by ultrastructural electron microscopy: a decreased number and irregular morphology of chondrocytes, chromatin margination and condensation, increased electron density and nucleus density, irregular nucleus morphology, nucleus condensation, nuclear changes and aggregation of matrix particles, uneven surface of the nucleus membrane, nucleolus coagulation, decreased cell size, cytoplasmic concentration, a slightly increased number and mild swelling of mitochondria, scattered and poorly identifiable organelles, presence of many vacuoles in the cytoplasm (cavitation), glycoprotein-rich matrix with a very little amount of collagen, matrix-depleted cytoplasm coagulated into small granules, and vacuolar mitochondria. Such changes were consistent with the manifestations of apoptosis, demonstrating excessive chondrocyte apoptosis in DDH-related acetabular dysplasia. The biomechanical changes of the cartilage may be one of the inducing factors for high chondrocyte apoptosis in early DDH. The excessive apoptosis may result in an increased acetabular index in DDH and may also play an important role in the future cartilage degeneration.

\section{Apoptosis and caspase-3}

With the discovery of a growing number of apoptosis-related factors, the mechanisms

Genetics and Molecular Research 15 (3): gmr.15036809 
of apoptosis appear to be more complex than originally proposed. Many studies have demonstrated that inhibition of caspase- 3 can slow chondrocyte apoptosis (Matsuo et al., 2001; Pelletier et al., 2000). In the present study, caspase-3 expression was found to be higher in articular cartilage with early DDH-related acetabular dysplasia compared with the control side, and was positively correlated with apoptosis. These findings suggest that caspase- 3 is indeed involved in articular cartilage apoptosis in DDH, and may play a role in DDH-related acetabular dysplasia and cartilage degeneration. It is possible that changes in mechanical stress, internal environment, and blood supply following DDH can initiate a caspase cascade in the acetabular cartilage, which then leads to excessive apoptosis, and directly affects the development or morphology of the acetabular cartilage. These factors result in acetabular cartilage degeneration and acetabular dysplasia. Based on physiological and pathological characteristics of caspase- 3 , it is inferred that caspase- 3 overexpression may be the cause of acetabular cartilage degeneration.

\section{Apoptosis and Bcl-2}

Bcl-2, short for B-cell lymphoma/leukemia-2, was the first apoptosis-related factor investigated (Feng et al., 1999). In the present study, immunohistochemical analysis revealed that the expression of Bcl-2 was relatively low in chondrocytes in acetabular dysplasia, which negatively correlated with cell apoptosis. This indicates that Bcl-2 expression may be inhibited during the acetabular chondrocyte apoptosis in $\mathrm{DDH}$, which may be due to functional inhibition. It is thereby proposed that low expression of $\mathrm{Bcl}-2$ in acetabular cartilage in DDH leads to reduced ability of cells to maintain the integrity of the mitochondrial membrane. This can be visualized by electron microscopy, where release of cytochrome $c$ and other apoptosis-inducing factor results in excessive apoptosis of acetabular chondrocytes. We conclude that this is one of the mechanisms by which Bcl-2 leads to acetabular cartilage degeneration in DDH.

\section{Relationship between caspase-3 and Bcl-2}

The present study found excessive apoptosis in acetabular cartilage in DDH, which was positively correlated with high caspase- 3 expression and low Bcl-2 expression. However, no significant difference was found in chondrocyte apoptosis and caspase- 3 or Bcl2 expression between the 8 - and 10-week experimental groups. These findings may indicate that excessive chondrocyte apoptosis plays an important role in the development of acetabular cartilage degeneration in DDH, but there are no significant changes in chondrocyte apoptosis and caspase- 3 or Bcl-2 expression with the increase in immobilization time and progression of cartilage degeneration. In this study, cartilage samples were obtained from an area that was found to bear the greatest stress during hip dislocation. Serious abrasion of acetabular cartilage in this area led to hyperplasia of acetabulum during adaptation to stress, thus resulting in a larger cartilage area that is easy to sample. Analysis of cartilage in other areas of the acetabulum was not carried out, and comparison of chondrocytes at different levels was not performed. Therefore, is it possible that apoptosis in acetabular cartilage with DDH in different areas may vary due to differential stress and apoptosis factors.

In conclusion, mechanisms for regulating apoptosis in acetabular cartilage with DDH are very complex, and many questions still remain. For example, what are the detailed mechanisms for the apoptotic pathways in DDH-related acetabular cartilage degeneration?

Genetics and Molecular Research 15 (3): gmr.15036809 
What are the other important factors involved in cartilage degeneration? How are they related to apoptosis? What is the relationship between caspase-3 and Bcl-2? Are there different stages of changes in apoptosis throughout the process of cartilage degeneration? What is the pattern and outcome of such changes? All these questions must be further explored in order to effectively develop a suitable therapeutic for this disease.

\section{Conflicts of interest}

The authors declare no conflict of interest.

\section{REFERENCES}

Feng L, Balakir R, Precht P and Horton Jr WE (1999). Bcl-2 regulates chondrocyte morphology and aggrecan gene expression independent of caspase activation and full apoptosis. J. Cell. Biochem. 74: 576-586. http://dx.doi. org/10.1002/(SICI)1097-4644(19990915)74:4<576::AID-JCB7>3.0.CO;2-N

Greenhill BJ, Hainau B, Ellis RD and el-Sayed RM (1995). Acetabular changes in an experimental model of developmental dysplasia of the hip (DDH). J. Pediatr. Orthop. 15: 789-793. http://dx.doi.org/10.1097/01241398-199511000-00012

Huang S, Zhao D and Yang L (2013). New approach to the treatment of adolescent hip dysplasia. Med. Hypothese 81: 122-124.http://dx.doi.org/10.1016/j.mehy.2013.04.005

Jiang RT, Yao CS, Bai JY and Hou Q (2014). Effects of Vam3 on sodium nitroprusside-induced apoptosis and SIRT1 and p53 expression in rat articular chondrocytes. Acta Pharmacol. Sin. 49: 608-614.

Liu S, Tian W, Wang J, Cheng L, et al. (2014). Two single-nucleotide polymorphisms in the DKK1 gene are associated with developmental dysplasia of the hip in the Chinese Han female population. Genet. Test. Mol. Biomarkers 18: 557-561.http://dx.doi.org/10.1089/gtmb.2014.0044

Matsuo M, Nishida K, Yoshida A, Murakami T, et al. (2001). Expression of caspase-3 and -9 relevant to cartilage destruction and chondrocyte apoptosis in human osteoarthritic cartilage. Acta Med. Okayama 55: 333-340.

Pelletier JP, Jovanovic DV, Lascau-Coman V, Fernandes JC, et al. (2000). Selective inhibition of inducible nitric oxide synthase reduces progression of experimental osteoarthritis in vivo: possible link with the reduction in chondrocyte apoptosis and caspase 3 level. Arthritis Rheumatol. 43: 1290-1299. http://dx.doi.org/10.1002/15290131(200006)43:6p<1290::AID-ANR11>3.0.CO;2-R

Genetics and Molecular Research 15 (3): gmr.15036809 